\title{
Kepler finds scores of planets around cool dwarf stars
}

NASA's rebooted mission, K2, seeks out new worlds closely orbiting stars smaller than the Sun.

\section{Ramin Skibba}

21 October 2016

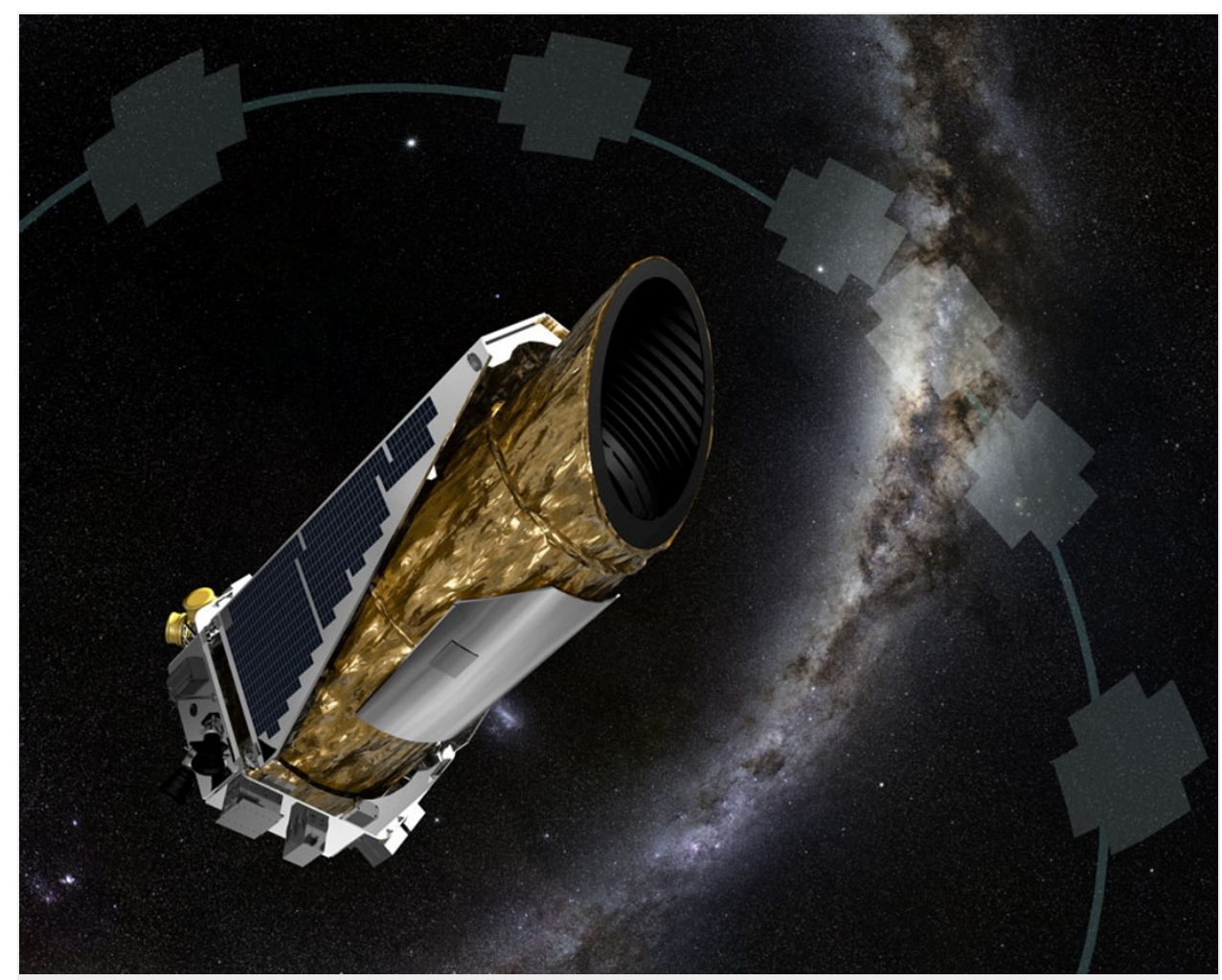

NASA Ames/JPL-Caltech/T Pyle

NASA's Kepler observatory has spotted 20 planets that orbit cool, small stars - the largest such haul so far. These long-lived stars, known as $\mathrm{K}$ and $\mathrm{M}$ dwarfs, are ubiquitous in the Milky Way and could turn out to host numerous habitable planets.

After the Kepler spacecraft experienced a mechanical failure in 2013 that made it impossible for it to keep observing its original targets, astronomers gave it a new mission, called K2. It now uses pressure from sunlight to help stabilize the craft. The latest observations with K2 revealed 87 planet candidates, on top of 667 previously announced candidates, almost all with sizes between those of Mars and Neptune.

Although the original Kepler mission examined many Sun-like stars, the majority of stars in our Galaxy are smaller, fainter, cooler stars, known as red dwarfs. Such stars make up nearly half the targets of the K2 mission. "There are more than 250 of them within 30 lightyears - all over the place — which is why some other astronomers here might call them the vermin of the sky," says Courtney Dressing, an astrophysicist at the California Institute of Technology in Pasadena who presented the research at a joint meeting of the American Astronomical Society's Division for Planetary Sciences and the European Planetary Science Congress in Pasadena on 19 October.

"Since these stars are the most common ones in the Galaxy, they help us learn how common life might be," says Victoria Meadows, an astronomer at the University of Washington in Seattle.

Of the confirmed planets, 63 are smaller than Neptune, and a few could be even smaller than Earth. But these small candidates remain to be confirmed. Dressing believes that these are probably "false positives" caused by other phenomena such as cosmic rays or an instrumental glitch.

Five of the confirmed planet candidates are in or near their star's 'habitable zone', the region that's neither too close to the star, nor 
too far from it, for life to arise. In our Solar System, the zone is roughly between the orbits of Venus and Mars.

Red dwarf stars give off less energy than larger, hotter stars, so their planets' habitable zones are closer in, often closer to their star than Mercury is to the Sun. Such planets transit frequently, some orbiting their star within just a few weeks, making it easier to use Kepler's instruments to detect the tell-tale dimming of stellar light.

The focus on red dwarfs stems partly from the K2 mission's constraints, which allow the astronomers less then three months to observe stars in its field of viewbefore having to rotate the craft. Moving from field to field poses a challenge, but it also gives the team an opportunity to investigate more objects. "It's fun to study a new set of stars every 80 days," Dressing says.

Dressing's research also paves the way for more sensitive future missions designed to look for Earth-sized planets, says Christa van Laerhoven, a planetary scientist at the Canadian Institute for Theoretical Astrophysics in Toronto. Such missions include NASA's Transiting Exoplanet Survey Satellite, scheduled to launch in December next year.

Nature | doi:10.1038/nature.2016.20853 